\title{
Adaptive LMS Filters for Cellular CDMA Overlay Situations
}

\author{
Jiangzhou Wang, Senior Member, IEEE, and Laurence B. Milstein, Fellow, IEEE
}

\begin{abstract}
This paper extends and complements previous research we have performed on the performance of nonadaptive narrowband suppression filters when used in cellular codedivision multiple-access (CDMA) overlay situations. In this paper, an adaptive least mean square (LMS) filter is applied to a cellular CDMA overlay in order to reject narrowband interference. An accurate expression for the steady-state tap-weight covariance matrix is derived for the real LMS algorithm for arbitrary statistics of the overlaid interference. Numerical results illustrate that when the ratio of the narrowband interference bandwidth to the spread spectrum bandwidth is small, the LMS filter is very effective in rejecting the narrowband interference. Furthermore, it is seen that the performance of the LMS filter in a CDMA overlay environment is not significantly worse than the performance of an ideal Wiener filter, assuming the LMS filter has had sufficient time to converge.
\end{abstract}

\section{INTRODUCTION}

A $\mathrm{S}$ is well known, a Wiener filter can provide vastly improved performance for a code-division multiple-access (CDMA) receiver operating in narrowband interference. For large narrowband interference, the CDMA system with a Wiener filter can support many more users than can the system without a filter.

However, in practice, since cellular CDMA users are mobile, there are Doppler frequency-shifts. Also, since the cellular channel is fading, the signal and interference statistics are rarely constant. Thus, the Wiener filter must be made adaptive. In this paper, we are concerned with the adaptive least mean square (LMS) filter, which is one of the simplest adaptive algorithms to analyze and implement. The work concentrates on the uplink, steady-state, performance of CDMA overlay systems with adaptive LMS filters, assuming convergence has been achieved. This work extends and complements the work of [1], which evaluates the bit-error rate (BER) performance of cellular CDMA overlay situations with Wiener filters.

The paper is organized as follows: Section II introduces the basic concepts and notation of the cellular CDMA system and describes the statistics of the misadjustment component of

Manuscript received April 29, 1995; revised November 22, 1995. This work was supported by the Office of Naval Research under Grant N00014-91-J1234 and the National Science Foundation Industry/University Cooperative Research Center on Ultra-High Speed Integrated Circuits and Systems at the University of California, San Diego.

J. Wang is with the Department of Electrical and Electronics Engineering. University of Hong Kong, Hong Kong.

L. B. Milstein is with the Department of Electrical and Computer Engineering, University of California at San Diego, La Jolla, CA 92093 USA.

Publisher Item Identifier S 0733-8716(96)05205-5. the adaptive LMS filter. Section III presents the performance analysis of the adaptive CDMA receiver. Numerical results are presented in Section IV, and Section $V$ provides the conclusions.

\section{Statistics of the MisaduUstment Filter}

A receiver operating in a cellular CDMA overlay environment and which incorporates the use of a Wiener filter was described in [1]. By replacing the Wiener filter with an adaptive LMS filter, the adaptive CDMA receiver is constituted. As in [2]-[4], the adaptive LMS filter is modelled as consisting of a Wiener filter and a misadjustment filter operating in parallel (see Fig. 1).

First, the basic concepts and notation of the cellular CDMA system are introduced. It is assumed that in the cellular system, there are $C$ cells, each of which contains $K$ active users and one base station. Therefore, there are $C K$ active users, for the entire cellular system. The cellular mobile channel between a mobile user and a base station is assumed to be a multipath Rician-fading channel, where there are $L$ paths associated with each user.

As shown in Fig. 1, the receiver consists of the following parts: a bandpass (BP) filter, an adaptive LMS filter, a DSdespreader, and a hard decision device. The input signal $r(t)$ to the adaptive filter is the sum of all CDMA signals, a narrowband binary phase shift keying (BPSK) representing the signal which is overlaid by the CDMA network, and band-limited additive white Gaussian noise (AWGN). That is

$$
\begin{aligned}
r(t)= & \operatorname{Re}\left\{\sqrt{2 P} \sum_{k=1}^{C K} \sqrt{\epsilon\left(\gamma, c_{k}, k\right) \sum_{l=1}^{L}\left[A_{k l} \exp \left(j \phi_{k l}\right)\right.}\right. \\
& \left.+\beta_{k l} \operatorname{cxp}\left(j \psi_{k l}\right)\right] b_{k}\left(t-\tau_{k l}\right) a_{k}\left(t-\tau_{k l}\right) \exp \left(j 2 \pi f_{0} t\right) \\
& \left.+\sqrt{2 J} d(t) \exp \left[j\left(2 \pi\left(f_{0}+\Delta\right) t+\theta\right)\right]\right\}+n(t)
\end{aligned}
$$

where $\gamma$ is a propagation exponent, and $c_{k}$ denotes the cell in which the $k$ th user is located; the users are numbered such that $c_{k}=\operatorname{int}[1+(k-1) / K]$, where int $[x]$ stands for integer part of $x$. The function $\epsilon\left(\gamma, c_{k}, k\right)$ represents the $\gamma$ th power of the ratio of the distance of the $k$ th user to its own base station ( $c_{k}$ th cell) to the distance of the $k$ th user to the first cell base station $\left(c_{k}=1\right)$. For the first cell (cell of interest), we assume $\left.\epsilon\left(\gamma, c_{k}, k\right)\right|_{c_{k}=1}=1$ because of perfect adaptive power control. The parameter $f_{0}$ denotes the CDMA carrier frequency, $b_{k}(t)$ is the $k$ th binary 


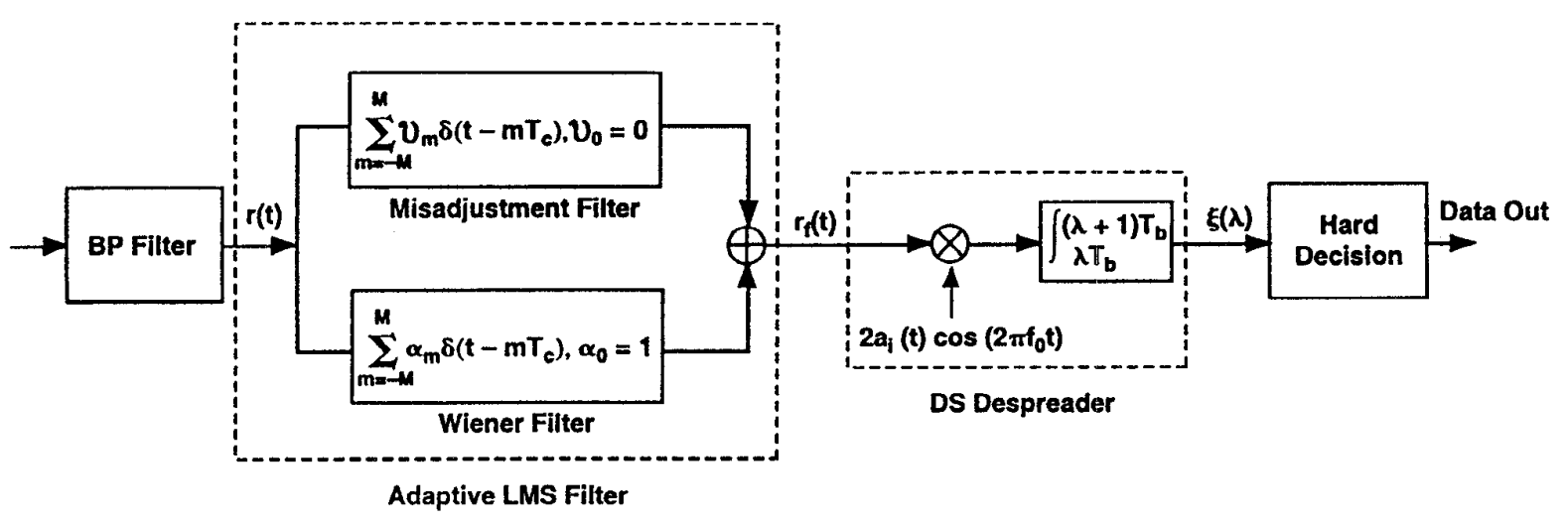

Fig. 1. An adaptive CDMA receiver model.

information sequence with bit duration $T_{b}, a_{k}(t)$ is a random spreading sequence with chip duration $T_{c}$ and processing gain $N\left(N=T_{b} / T_{c}\right)$, and $A_{k l}\left(0 \leq A_{k l} \leq 1\right)$ and $\phi_{k l}$ are the gain and phase of the specular component of the $l$ th path from the $k$ th user, respectively. It is assumed that $A_{k l}=A$ for all $k$ and $l$, and $\phi_{k l}$ is uniformly distributed in $[0,2 \pi]$. The random gain $\beta_{k l}$ and phase $\psi_{k l}$ of the fading component of the $l$ th path of the $k$ th user have a Rayleigh distribution with $E\left[\beta_{k l}^{2}\right]=2 \rho_{k l}=2 \rho$ for all $k$ and $l$, and a uniform distribution in $[0,2 \pi]$, respectively. The path delay, $\tau_{k l}$, is uniformly distributed in $\left[0, T_{b}\right]$ and, to simplify some of the analysis to follow, we assume $\left|\tau_{k l}-\tau_{k \hat{l}}\right| \geq T_{c}$ for $l \neq \hat{l}$. The gains, delays and phases of different paths and/or of different users are assumed to be statistically independent. Furthermore, $J$ and $\theta$ denote the received nonfading BPSK narrowband interference power and phase, respectively, $\Delta$ stands for the frequency offset of the interference, and $d(t)$ is the binary data sequence of the narrowband interference, $J(t)$, having bit duration $T_{j}$. The two parameters $p$ and $q$ are defined as the ratio of the interference bandwidth to the spread bandwidth and the ratio of the offset of the interference carrier frequency to half of the spread bandwidth, respectively, (i.e., $p=T_{c} / T_{j}$ and $\left.q=\Delta T_{c}\right)$. Finally, $n(t)$ is bandlimited AWGN with twosided power spectral density $N_{0} / 2$ and bandwidth $2 T_{c}^{-1}$. Note that, for simplicity, while we have bandlimited the noise, we have assumed that the BPF passes the signal undistorted.

The autocorrelation function of the input signal is defined by

$$
\rho_{r}(\tau)=E[r(t) r(t+\tau)]
$$

although only values of $\rho_{r}(\tau)$ at $\tau=m T_{c}$, where $m$ is an integer, are needed. In particular, $\rho_{r}\left(m T_{c}\right)$ can be written as

$$
\rho_{r}\left(m T_{c}\right)=\rho_{s}\left(m T_{c}\right)+\rho_{j}\left(m T_{c}\right)+\rho_{n}\left(m T_{c}\right)
$$

where $\rho_{s}\left(m T_{c}\right), \rho_{j}\left(m T_{c}\right)$ and $\rho_{n}\left(m T_{c}\right)$ correspond to, respectively, the CDMA signals, the interference and the noise. Assuming that $f_{0} T_{c}$ is an integer, the CDMA term is given by

$$
\begin{aligned}
\rho_{s}\left(m T_{c}\right)= & E\left\{\left[\sqrt { 2 P } \sum _ { k _ { 1 } = 1 } ^ { C K } \sqrt { \epsilon ( \gamma , c _ { k _ { 1 } } , k _ { 1 } ) } \sum _ { l _ { 1 } = 1 } ^ { L } \left[A_{k_{1} l_{1}} \cos \phi_{k_{1} l_{1}}\right.\right.\right. \\
& \left.\left.+\beta_{k_{1} l_{1}} \cos \psi_{k_{1} l_{1}}\right] b_{k_{1}}\left(t-\tau_{k_{1} l_{1}}\right) a_{k_{1}}\left(t-\tau_{k_{1} l_{1}}\right)\right]
\end{aligned}
$$

$$
\begin{aligned}
& {\left[\sqrt{2 P} \sum_{k_{2}=1}^{C K} \sqrt{\epsilon\left(\gamma, c_{k_{2}}, k_{2}\right)}\right.} \\
\cdot & \sum_{l_{2}=1}^{L}\left[A_{k_{2} l_{2}} \cos \phi_{k_{2} l_{2}}+\beta_{k_{2} l_{2}} \cos \psi_{k_{2} l_{2}}\right] \\
\cdot & \left.\left.b_{k_{2}}\left(t+m T_{c}-\tau_{k_{2} l_{2}}\right) a_{k_{2}}\left(t+m T_{c}-\tau_{k_{2} l_{2}}\right)\right]\right\} \\
= & 2 P \sum_{k_{1}=1}^{C K} \sum_{k_{2}=1}^{C K} E\left[\sqrt{\epsilon\left(\gamma, c_{k_{1}}, k_{1}\right)} \sqrt{\epsilon\left(\gamma, c_{k_{2}, k_{2}}\right)}\right] \\
& \cdot \sum_{l_{1}=1}^{L} \sum_{l_{2}=2}^{L}\left\{E \left[\left(A_{k_{1} l_{1}} \cos \phi_{k_{1} l_{1}}+\beta_{k_{1} l_{1}} \cos \psi_{k_{1} l_{1}}\right)\right.\right. \\
& \left.\cdot\left(A_{k_{2} l_{2}} \cos \phi_{k_{2} l_{2}}+\beta_{k_{2} l_{2}} \cos \psi_{k_{2} l_{2}}\right)\right] \\
& \cdot E\left[b_{k_{1}}\left(t-\tau_{k_{1} l_{1}}\right) b_{k_{2}}\left(t+m T_{c}-\tau_{k_{2} l_{2}}\right)\right] \\
& \left.\cdot E\left[a_{k_{1}}\left(t-\tau_{k_{2} l_{2}}\right) a_{k_{2}}\left(t+m T_{c}-\tau_{k_{2} l_{2}}\right)\right]\right\}
\end{aligned}
$$

where $E\left[a_{k_{1}}\left(t-\tau_{k_{1} l_{1}}\right) a_{k_{2}}\left(t+m T_{c}-\tau_{k_{2} l_{2}}\right)\right]=0$ for $k_{1} \neq k_{2}$ and $E\left(\cos \phi_{k l_{1}} \cos \phi_{k l_{2}}\right)=E\left(\cos \psi_{k l_{1}} \cos \psi_{k l_{2}}\right)=0$ for $l_{1} \neq l_{2}$. Therefore, $\rho_{s}\left(m T_{c}\right)$ reduces to

$$
\begin{aligned}
\rho_{s}\left(m T_{c}\right)= & P \sum_{k=1}^{C K} E\left[\epsilon\left(\gamma, c_{k}, k\right)\right] \sum_{l=1}^{L}\left(A^{2}+2 \rho\right) \\
& \cdot E\left[b_{k}\left(t-\tau_{k l}\right) b_{k}\left(t+m T_{c}-\tau_{k l}\right)\right] \\
& \cdot E\left[a_{k}\left(t-\tau_{k l}\right) a_{k}\left(t+m T_{c}-\tau_{k l}\right)\right]
\end{aligned}
$$

Since

$$
E\left[a_{k}\left(t-\tau_{k l}\right) a_{k}\left(t+m T_{c}-\tau_{k l}\right)\right]=\delta(m)
$$

with a similar expression for the autocorrelation of $b_{k}(t)$, we have

$$
\rho_{s}\left(m T_{c}\right)=P\left(A^{2}+2 \rho\right) L \delta(m) \sum_{k=1}^{C K} E\left[\epsilon\left(\gamma, c_{k}, k\right)\right]
$$

where $\delta(m)$ is the Kronecker delta function, and $E\left[\epsilon\left(\gamma, c_{k}\right.\right.$, $k)]=\epsilon\left(\gamma, c_{k}\right)$ is independent of the position of the $k$ th user since $E\left[\epsilon\left(\gamma, c_{k}, k\right)\right]$ is the average of the $k$ th user's position over the $c_{k}$ th cell. Considering a three-layer cellular model, 
we have

$$
\sum_{k=1}^{C K} E\left[\epsilon\left(\gamma, c_{k}, k\right)\right] \approx[1+\zeta(\gamma)] K
$$

where $\zeta(\gamma)$ represents the relative interference from all surrounding cells, and is defined as $\zeta(\gamma)=\sum_{c=2}^{C} \epsilon(\gamma, c)$. Therefore

$$
\rho_{s}\left(m T_{c}\right) \approx P\left(A^{2}+2 \rho\right)[1+\zeta(\gamma)] K L \delta(m) .
$$

The narrowband interference autocorrelation is given by

$$
\begin{aligned}
\rho_{j}\left(m T_{c}\right) & =\operatorname{Jrect}\left(1-\frac{|m| T_{c}}{T_{j}}\right) \cos \left(2 \pi \Delta m T_{c}\right) \\
& =J \operatorname{rect}(1-|m| p) \cos (2 \pi m q)
\end{aligned}
$$

where $\operatorname{rect}(x)=x$ or 0 for $x \geq 0$ or $x<0$, respectively. Also, the noise autocorrelation is given by

$$
\rho_{n}\left(m T_{c}\right)=2 N_{0} T_{c} \delta(m)
$$

so that, using (3)-(7), $\rho_{r}\left(m T_{c}\right)$ is given by

$$
\begin{aligned}
\rho_{r}\left(m T_{c}\right)= & P A^{2}\left\{\left(1+\frac{1}{H}\right) K L[1+\zeta(\gamma)] \delta(m)\right. \\
& +\frac{J}{S} \operatorname{rect}(1-|m| p) \cos (2 \pi m q) \\
& \left.+2 N\left[\frac{E_{b}}{N_{0}}\right]^{-1} \delta(m)\right\}
\end{aligned}
$$

where $H=A_{i l}^{2} /\left(2 \rho_{i l}\right)=A^{2} /(2 \rho)$ stands for the ratio of the specular component power to the fading component power, $J / S=J /\left(P A^{2}\right)$ is defined as the narrowband interference power-to-signal power ratio, and $E_{b} / N_{0}=P A^{2} T_{b} / N_{0}$ stands for the signal-to-noise ratio (SNR).

The adaptive filter output is given by

$$
r_{f}(t)=\sum_{m=-M}^{M}\left(\alpha_{m}+v_{m}\right) r\left(t-m T_{c}\right)
$$

where $\alpha_{m}, m=-M, \cdots, M$, denotes the $m$ th tap weight of the Wiener filter, and $v_{m}, m=-M, \cdots, M$, denotes the $m$ th steady-state tap-weight of the misadjustment filter. Note that $v_{0}$ is always zero, because the center tap of the Weiner filter is fixed at one (i.e., $\alpha_{0}=1$ ).

Most often, via a central limit theorem, it is argued that the steady-state tap weights of the misadjustment filter are jointly Gaussian [4] for small enough adaptation step size. Hence, with the joint Gaussian assumption, the tap-weight covariance matrix completely defines the statistics of the misadjustment filter. In Appendix A, it is shown that when it is assumed that the sum of all active CDMA signals is Gaussian, the steady state covariance matrix of the tap weight vector can be obtained (approximately) by solving (A18), reproduced below as $(10)$

$$
\begin{aligned}
\sum_{n_{1}=1}^{2 M} & {\left[\left(R_{r}\right)_{m_{1} n_{1}}\left(R_{v}\right)_{n_{1} m_{2}}+\left(R_{v}\right)_{m_{1} n_{1}}\left(R_{r}\right)_{n_{1} m_{2}}\right] } \\
\approx & 2 \mu E\left[\left(e^{*}\right)^{2}\right]\left(R_{r}\right)_{m_{1} m_{2}}+2 \mu E\left[\left(e_{j}^{*}\right)^{2} j_{m_{1}} j_{m_{2}}\right] \\
& -2 \mu E\left[\left(e_{j}^{*}\right)^{2}\right] E\left(j_{m_{1}} j_{m_{2}}\right), \quad m_{1}, m_{2}=1, \cdots, 2 M
\end{aligned}
$$

where $R_{r}$ and $R_{v}$ are the covariance matrices of the input signal sample vector and the tap weights of the misadjustment filter, respectively, and $(R)_{m_{1} n_{1}}$ denotes the $m_{1}$ th row and $n_{1}$ th column element of $R$. Note that $\left(R_{r}\right)_{m_{1} m_{2}}=\rho_{r}\left[\left(m_{1}-\right.\right.$ $\left.m_{2}\right) T_{c}$ ], given by (8), $\mu$ is the adaptation step size, and $e^{*}$ and $e_{j}^{*}$ are the Wiener prediction error for the composite input signal and from the narrowband component of the input, respectively, and are defined as $e^{*}(*)=\sum_{m=-M}^{M} \alpha_{m} r(t-$ $\left.m T_{c}\right)$ and $e^{*}(*)_{j}=\sum_{m=-M}^{M} \alpha_{m} J\left(t-m T_{c}\right)$, respectively, where $J(t)$ is the narrowband component of the input. In the special case where the narrowband interference, $j_{m}$, at the $m$ th tap [see (A14)], is Gaussian, $e_{j}^{*}$ is independent of $j_{m}$. Therefore, the last two terms of (10) cancel each other, so that (10) reduces to the well-known form (A13). Analogous to the derivation of $\rho_{r}\left(m T_{c}\right), E\left[\left(e^{*}\right)^{2}\right]$ are $E\left[\left(e_{j}^{*}\right)^{2}\right]$ are given by

$$
\begin{aligned}
E\left[\left(e^{*}\right)^{2}\right]= & E\left[\sum_{m=-M}^{M} \alpha_{m} r\left(t-m T_{c}\right)\right]^{2} \\
\approx & P A^{2}\left\{\left(1+\frac{1}{H}\right) K L[1+\zeta(\gamma)] \sum_{m_{1}=-M}^{M} \alpha_{m}^{2}\right. \\
& +\frac{J}{S} \sum_{m_{1}=-M_{2}=-M}^{M} \sum_{m_{1}} \alpha_{m_{2}} \\
& \cdot \cos \left[2 \pi\left(m_{1}-m_{2}\right) q\right] \\
& \cdot \operatorname{rect}\left(1-\left|m_{1}-m_{2}\right| p\right)+2 N\left(E_{b} / N_{0}\right)^{-1} \\
& \left.\cdot \sum_{m=-M}^{M} \alpha_{m}^{2}\right\} \\
\approx & P A^{2}\left\{\left(1+\frac{1}{H}\right) K L[1+\zeta(\gamma)] \sum_{m=-M}^{M} \alpha_{m}^{2}\right. \\
& \left.+2 N\left(E_{b} / N_{0}\right)^{-1} \sum_{m=-M}^{M} \alpha_{m}^{2}\right\}+E\left[\left(e_{j}^{*}\right)^{2}\right]
\end{aligned}
$$

where it is assumed that $f_{0} T_{c}$ is an integer, and

$$
\begin{aligned}
E\left[\left(e_{j}^{*}\right)^{2}\right]= & P A^{2} \frac{J}{S} \sum_{m_{1}=-M}^{M} \sum_{m_{2}=-M}^{M} \alpha_{m_{1}} \alpha_{m_{2}} \\
& \cdot \operatorname{rect}\left(1-\left|m_{1}-m_{2}\right| p\right) \cos \left[2 \pi\left(m_{1}-m_{2}\right) q\right] .
\end{aligned}
$$

\section{PERFORMANCE ANALysis OF THE ADAPTIVE CDMA RECEIVER}

Assuming the $l$ th path of the $i$ th user (reference user) of the cell-of-interest is the reference path, and $\tau_{i l}=\phi_{i l}=0$, the despreader output is given by

$$
\xi(\lambda)=\int_{\lambda T_{b}}^{(\lambda+1) T_{b}} r_{f}(t) 2 a_{i}(t) \cos \left(2 \pi f_{0} t\right) d t .
$$

To simplify the analysis, the self-interference, due to the main path of the reference user, and caused by the taps of the filter excluding the zeroth tap, is neglected when the number of active users is much greater than unity, as in [1]. Therefore, 
(12) reduces to

$$
\xi(\lambda) \approx \sqrt{2 P} A_{i l} T_{b} b_{i}^{(\lambda)}+D(\lambda)+\sum_{k=1}^{C K} I_{k}+N(\lambda)+J(\lambda) .
$$

1) The first term is the desired signal, corresponding to the specular component of the $l$ th path of the reference user and the zeroth tap of the Wiener filter. The useful signal power is $S_{T}=2 P A^{2} T_{b}^{2}$.

2) $D(\lambda)$ is an interference term with conditional variance $\sigma_{D}^{2}=2 P \rho T_{b}^{2}$ due to the fading component of the $l$ th path of the reference user and the zeroth tap of the Wiener filter (see [1]).

3) $I_{k}$ is either a multipath interference term for $k=i$, or an in-cell multiple access interference term for $k=$ $1, \cdots, K, k \neq i$, or an adjacent cell multiple access interference term for $k=K+1, \cdots, C K$, and is given by

$$
I_{k}=\left\{\begin{array}{l}
\sum_{\substack{\hat{i}=1 \\
i \neq l}}^{L} I_{i, \hat{l}}, \quad k=i, \\
\text { multipath interference } \\
\sum_{\hat{l}=1}^{L} I_{k, \hat{l}}, \quad k=1, \cdots, K, k \neq i, \\
\quad \text { in-cell multiple-access interference } \\
\sum_{\hat{l}=1}^{L} I_{k, \hat{l}}, \quad k=K+1, \cdots, C K, \\
\text { adjacent-cell multi-access interference. }
\end{array}\right.
$$

For a large number of CDMA users $(K \gg 1)$, the effect of the multipath of the desired user is very small, since it roughly acts as one additional user. However, its inclusion in the analysis greatly complicates that analysis, and so it will be ignored, as justified in [1]. Then, in (14), for $k \neq i$

$$
\begin{aligned}
I_{k, \hat{l}}= & \sqrt{2 P}\left[A_{k \hat{l}} \cos \left(\phi_{k \hat{l}}\right)+\beta_{k \hat{l}} \cos \left(\psi_{k \hat{l}}\right)\right] \\
& \cdot \sqrt{\epsilon\left(\gamma, c_{k}, k\right)} \sum_{m=-M}^{M}\left(\alpha_{m}+v_{m}\right) \\
& \cdot \int_{\lambda T_{b}}^{(\lambda+1) T_{b}} b_{k}\left(t-\tau_{k \hat{l}}-m T_{c}\right) a_{k}\left(t-\tau_{k \hat{l}}-m T_{c}\right) \\
& \cdot a_{i}(t) d t .
\end{aligned}
$$

The conditional variance of the total in-cell multiple access interference and adjacent cell multiple access interference terms $\left(\sum_{\substack{k=1 \\ k \neq i}}^{C K} I_{k}\right)$ equals (see Appendix B), conditioned on the tap weights $\left(v \equiv\left[v_{-M}, \cdots, v_{M}\right]\right)$ of the misadjustment filter and the spreading sequence $\left\{a_{j}^{(i)}\right\}$ of the reference user (the $i$ th user)

$$
\begin{aligned}
\sigma_{0}^{2}\left(v, a_{i}(t)\right) & \sigma_{0}^{2}(v)=E\left[\left(\sum_{\substack{k=1 \\
k \neq i}}^{C K} I_{k}\right)^{2} \mid\left\{v_{m}\right\},\left\{a_{j}^{(i)}\right\}\right] \\
\cdot & 4[(1+\zeta(\gamma)) K L-L] P\left(\frac{A^{2}}{2}+\rho\right) T_{b}^{2} \\
& \cdot\left[\sum_{m=-M}^{M}\left(\alpha_{m}+v_{m}\right)^{2}\right.
\end{aligned}
$$

$$
\left.+\frac{1}{2} \sum_{m=-M}^{M}\left(\alpha_{m}+v_{m}\right)\left(\alpha_{m+1}+v_{m+1}\right)\right] /(3 N)
$$

4) $N(\lambda)$ is due to the thermal noise; its conditional variance equals $\sigma_{N}^{2}=N_{0} T_{b} \sum_{m=-M}^{M}\left(\alpha_{m}+v_{m}\right)^{2}$.

5) $J(\lambda)$ is due to the BPSK narrowband interference, and is given by

$$
\begin{aligned}
J(\lambda)= & \sum_{m=-M}^{M}\left(\alpha_{m}+v_{m}\right) \\
& \cdot \int_{\lambda T_{b}}^{(\lambda+1) T_{b}} \sqrt{2 J} d\left(t-m T_{c}\right) \\
& \cdot \cos \left[2 \pi \Delta\left(t-m T_{c}\right)+\theta\right] a_{i}(t) d t .
\end{aligned}
$$

In order to simply analyze the statistics of the narrowband interference term, assume that there are an integer number of bits of the narrowband waveform in $T_{b}$ seconds, and ignore any timing offset between the bits of the narrowband BPSK signal and the bits of the reference CDMA signal. Then, as in [1], (17) can be approximated (for $n>>m$ ) as

$$
\begin{aligned}
J(\lambda)= & \sqrt{2 J} T_{c} \sum_{m=-M}^{M}\left(\alpha_{m}+v_{m}\right) \sum_{n=0}^{N-1} a_{n}^{(i)} d_{[p(n-m)]} \\
& \cdot \beta(n, m, \theta) \\
= & \sqrt{2 J} T_{c} \sum_{n=0}^{N-1} \sum_{m=-M}^{M}\left(\alpha_{m}+v_{m}\right) a_{n}^{(i)} d_{[p(n-m)]} \\
& \cdot \beta(n, m, \theta)
\end{aligned}
$$

where

$$
\begin{aligned}
\beta(n, & m, \theta) \\
& \triangleq \frac{\sin [2 \pi q(n-m+1)+\theta]-\sin [2 \pi q(n-m)+\theta]}{2 \pi q} \\
& =\frac{\sin (\pi q) \cos [2 \pi q(n-m)+\theta]}{\pi q}
\end{aligned}
$$

$\left\{d_{[p(n-m)]}\right\}$ is the data sequence of the narrowband waveform, and $[x]$ is the integer portion of $x$. Note that (18) can be rewritten as

$$
J(\lambda)=\sqrt{2 J} T_{c} \sum_{n=0}^{N-1} x_{n}
$$

where

$$
x_{n}=\sum_{m=-M}^{M}\left(\alpha_{m}+v_{m}\right) a_{n}^{(i)} d_{[p(n-m)]} \beta(n, m, \theta) .
$$

Conditioned on $\left\{v_{m}\right\}$ and $\theta, x_{n}$ is a function of $\left\{d_{[p(n-m)]}\right\}$ and $\left\{a_{n}^{(i)}\right\}$. As in [1], it can be shown that the sequence $\left\{x_{n}\right\}$ is, conditionally, a $2 M$-dependent sequence. As $N \rightarrow \infty$, the sequence $\left\{x_{n}\right\}$ satisfies the conditions of the Hoeffding-Robbins version of the central limit theorem for dependent random variables, given $\left\{v_{m}\right\}$ and $\theta$. 
If we denote the conditional variance of $J(\lambda)$ by $\sigma_{j}^{2}(\theta, v)$, conditioned on $\theta$ and $v$, then

$$
\begin{aligned}
\sigma_{j}^{2}(\theta, v) & \\
= & 2\left(T_{b}^{2} / N^{2}\right) \\
& \cdot \sum_{n=0}^{N-1} \sum_{m_{1}=-M}^{M} \sum_{m_{2}=-M}^{M}\left(\alpha_{m_{1}}+v_{m_{1}}\right)\left(\alpha_{m_{2}}+v_{m_{2}}\right) \\
& \cdot \beta\left(n, m_{1}, \theta\right) \beta\left(n, m_{2}, \theta\right) \delta\left(\left[p\left(n-m_{1}\right)\right],\left[p\left(n-m_{2}\right)\right]\right)
\end{aligned}
$$

where $\delta(\cdot, \cdot)$ is the Kronecker delta function.

Assuming that the sum of the in cell multiple access interference and adjacent cell interference is conditionally Gaussian, the decision variable $\xi(\lambda)$ in (13) is conditionally Gaussian, conditioned on the random phase, $\theta$, of the narrowband interference, the random tap-weight variables, $v$, of the misadjustment filter, and the spreading sequence, $a_{i}(t)$, of the reference user. However, the conditional variances in (16) and (22) are not functions of $a_{i}(t)$. Therefore, the bit-error rate (BER) is given by

$$
P_{e}=\frac{1}{2 \pi} \int_{0}^{2 \pi} \int_{-\infty}^{\infty} \cdots \int_{-\infty}^{\infty} P_{e}(\theta, v) P(v) d \theta d v_{-M} \cdots d v_{M}
$$

where $P(v)$ is a jointly Gaussian distribution, given by

$$
P(v)=\frac{1}{(2 \pi)^{M}\left[\operatorname{det}\left(R_{v}\right)\right]^{\frac{1}{2}}} \exp \left[-\frac{1}{2} v R_{v}^{-1} v^{T}\right]
$$

and where $R_{v}$ is the steady-state tap weight covariance matrix of $v, \operatorname{det}(\cdot)$ is its determinant, $R_{v}^{-1}$ denotes the inverse of $R_{v}$, and $v^{T}$ denotes the transpose of $v$.

In (23), $P_{e}(\theta, v)$ is given by

$$
P_{e}(\theta, v)=\frac{1}{\sqrt{2 \pi}} \int_{\sqrt{\operatorname{SNR}(\theta, v)}}^{\infty} e^{-x^{2} / 2} d x
$$

where $\operatorname{SNR}(\theta, v)$ is the total $\operatorname{SNR}$, conditioned on $\theta$ and $v$, given by

$$
\begin{aligned}
\operatorname{SNR}(\theta, v)= & \frac{1}{2} \cdot \frac{S_{T}}{\sigma_{N}^{2}+\sigma_{D}^{2}+\sigma_{0}^{2}(v)+\sigma_{j}^{2}(\theta, v)} \\
= & \left\{\left(\frac{E_{b}}{N_{0}}\right)^{-1} \sum_{m=-M}^{M}\left(\alpha_{m}+v_{m}\right)^{2}+\frac{1}{H}\right. \\
& +2\left(1+\frac{1}{H}\right) \frac{[(1+\zeta(\gamma)) K L-L]}{3 N} \\
& \cdot\left[\sum_{m=-M}^{M}\left(\alpha_{m}+v_{m}\right)^{2}\right. \\
& \left.+\frac{1}{2} \sum_{m=-M}^{M}\left(\alpha_{m}+v_{m}\right)\left(\alpha_{m+1}+v_{m+1}\right)\right] \\
& \left.+\frac{2(J / S)}{N^{2}} \cdot \sum_{n=0}^{N-1} \sum_{m_{1}=-M m_{2}=-M}^{M} \sum_{m_{1}}^{M}+v_{m_{1}}\right) \\
& \cdot\left(\alpha_{m_{2}}+v_{m_{2}}\right) \beta\left(n, m_{1}, \theta\right) \beta\left(n, m_{2}, \theta\right) \\
& \left.\cdot \delta\left(\left[p\left(n-m_{1}\right)\right],\left[p\left(n-m_{2}\right)\right]\right)\right\}^{-1}
\end{aligned}
$$

and where $E_{b}=P A^{2} T_{b}$ is the average energy per bit, $J / S=J /\left(P A^{2}\right)$ denotes the interference interference power to useful signal power ratio, and $H=A^{2} /(2 \rho)$.

\section{NUMERICAL RESULTS}

Unless otherwise noted, the numerical results for the BER's of the adaptive CDMA overlay system are presented for the following common parameters: It is assumed that the ratio of the interference bandwidth to the spread spectrum bandwidth is $10 \%(\rho=0.1)$ and the ratio of the offset of the interference carrier frequency to half of the spread spectrum bandwidth is either zero or $20 \%(q=0,0.2)$. The processing gain and the number of taps on each side of a suppression filter are set at $N=255$ and $M=2$, respectively. The number of paths, the propagation exponent and the ratio of the specular component power to the fading component power are assumed to be $L=3, \gamma=3$, and $H=7 \mathrm{~dB}$, respectively. Note that $\gamma=3$ means that the adjacent cell interference $\zeta(r)=0.97$. That is, the interference from all adjacent cells is $97 \%$ of the interference of the cell-of-interest. Finally, the adaptation step size is selected as

$$
\mu=\frac{1}{10 \lambda_{\max }} \leq \frac{1}{20 M \rho_{r}(0)}
$$

where $\lambda_{\max }$ and $\rho_{r}(0)$ are the maximum eigenvalue of the covariance matrix of the input signal and the power of the input signal, respectively.

Fig. 2 illustrates the BER's of the adaptive CDMA overlay system as a function of $E_{b} / N_{0}$. Fig. 2(a) and (b) correspond to $q=0$ and $q=0.2$, respectively. It is seen that, as expected, the adaptive LMS filter is very effective in rejecting the narrowband interference. Note that for $q=0$, the adaptive filter is not as effective as it is for $q=0.2$, compared to the Wiener filter.

Fig. 3 shows the asymptotic $\left(E_{b} / N_{0} \rightarrow \infty\right)$ BER's of the CDMA system as a function of $(J / S) /[(1+\zeta(\gamma)) K L]$, which is roughly the ratio of $J / S$ to all multiple access interference. When $J / S$ is sufficiently small (i.e., $(J / S) /[(1+\zeta(\gamma)) K L] \ll$ $0 \mathrm{~dB}$ ), neither a Wiener filter nor an adaptive filter is necessary, because the multiple access interference dominates, whereas when $J / S$ is sufficiently large, an adaptive filter can provide a large improvement in performance.

In Fig. 4, the asymptotic BER of the CDMA overlay system is plotted as a function of the number of the active users $K$ per cell, for various values of $J / S$. It is clearly seen that the system using a suppression filter can support many more users than can the system without a filter.

In Fig. 5, the asymptotic BER of the CDMA overlay system is shown as a function of the ratio $(p)$ of the narrowband bandwidth to the spread spectrum bandwidth for $q=0,0.1,0.2$, and 0.4 . It is seen that when $p$ is small, an adaptive filter is very effective.

Fig. 6 illustrates the BER performance of the overlay system as a function of the ratio $(q)$ of the offset of the interference carrier frequency to the half spread spectrum bandwidth for different numbers of taps on each side. It is seen that when $q$ is small, the adaptive filter performs noticeably worse than the Wiener filter. However, when $q$ is large, the BER's of the 
BER

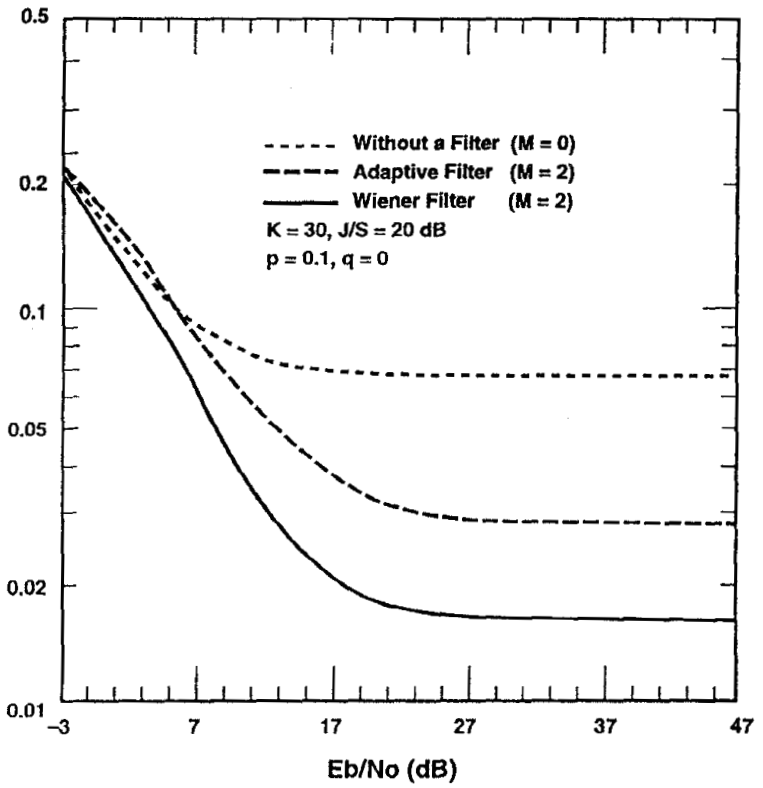

(a)

BER

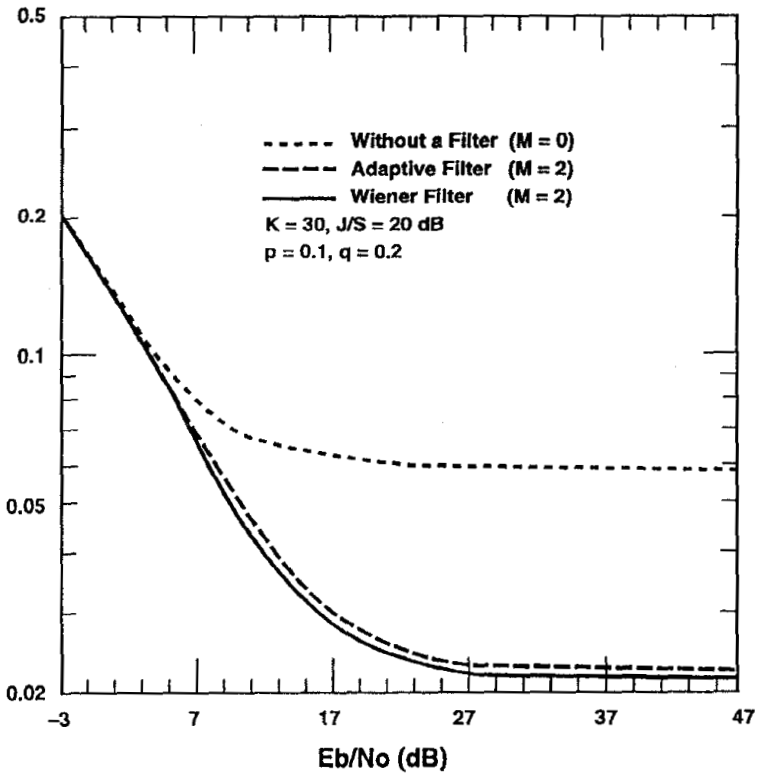

(b)

Fig. 2. BER performance of the CDMA overlay system versus $E_{b} / N_{0}$ : (a) $q=0$ and (b) $q=0.2$.

systems with the adaptive filter and the Wiener filter are almost identical. That is, the noise caused by the misadjustment filter is negligible.

\section{CONCLUSION}

In this paper, the effect of an adaptive LMS filter in a cellular CDMA overlay situation is investigated. An accurate

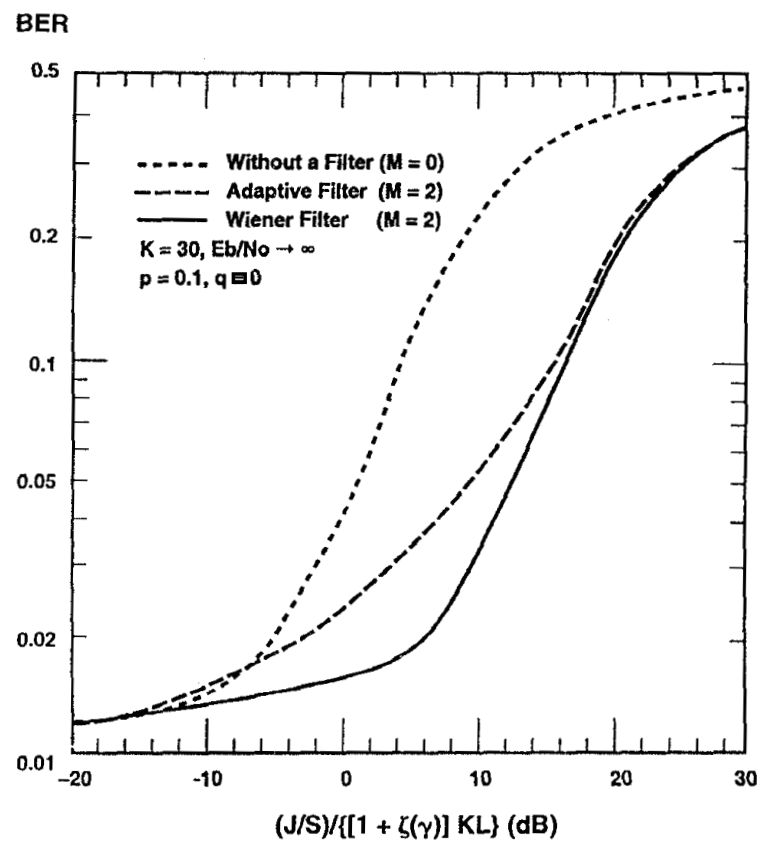

(a)

BER

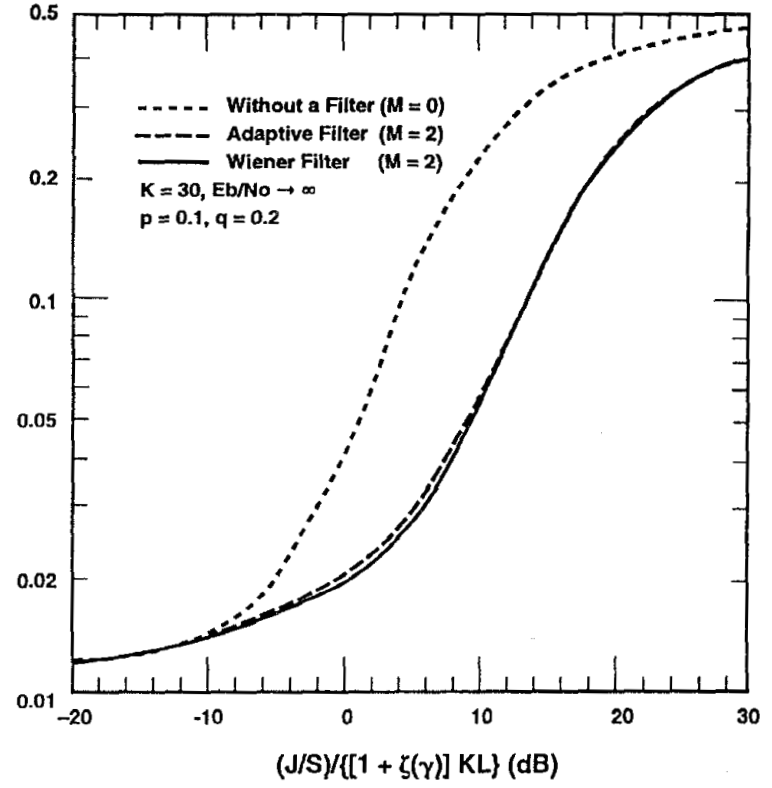

(b)

Fig. 3. The asymptotic BER of CDMA overlay system as a function of $(J / S) /[(1+\zeta(\gamma)) K L]:$ (a) $q=0$ and (b) $q=0.2$.

expression for the steady-state tap-weight covariance matrix is derived for the real LMS algorithm for arbitrary narrowband interference. It is shown that the adaptive filter is very effective in rejecting the narrowband interference when the ratio of the narrowband interference bandwidth to the spread spectrum bandwidth is small. Also, it is seen that the performance of the LMS filter in a CDMA overlay environment is not 
BER

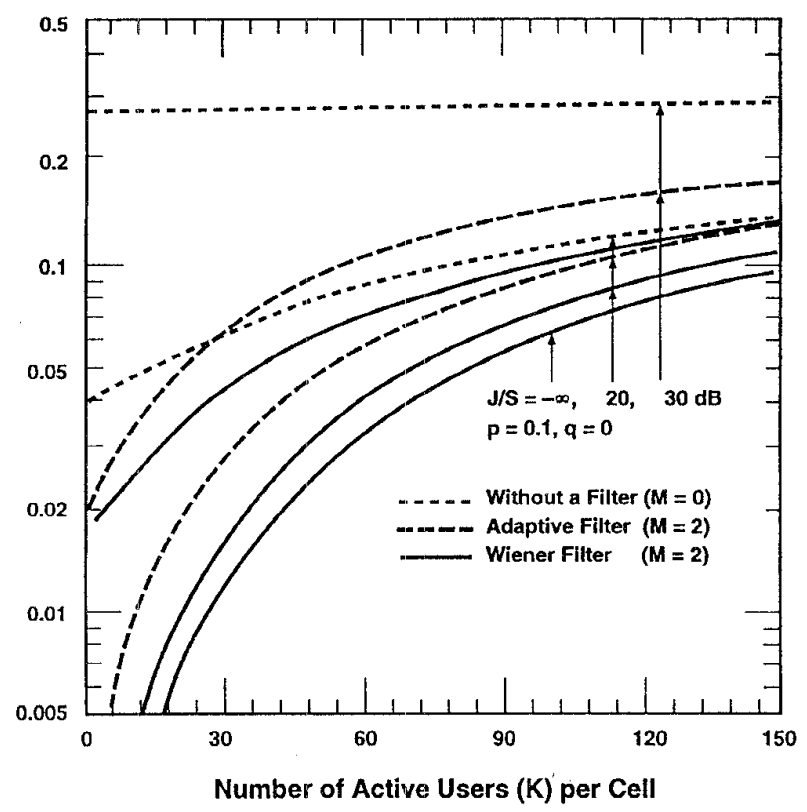

(a)

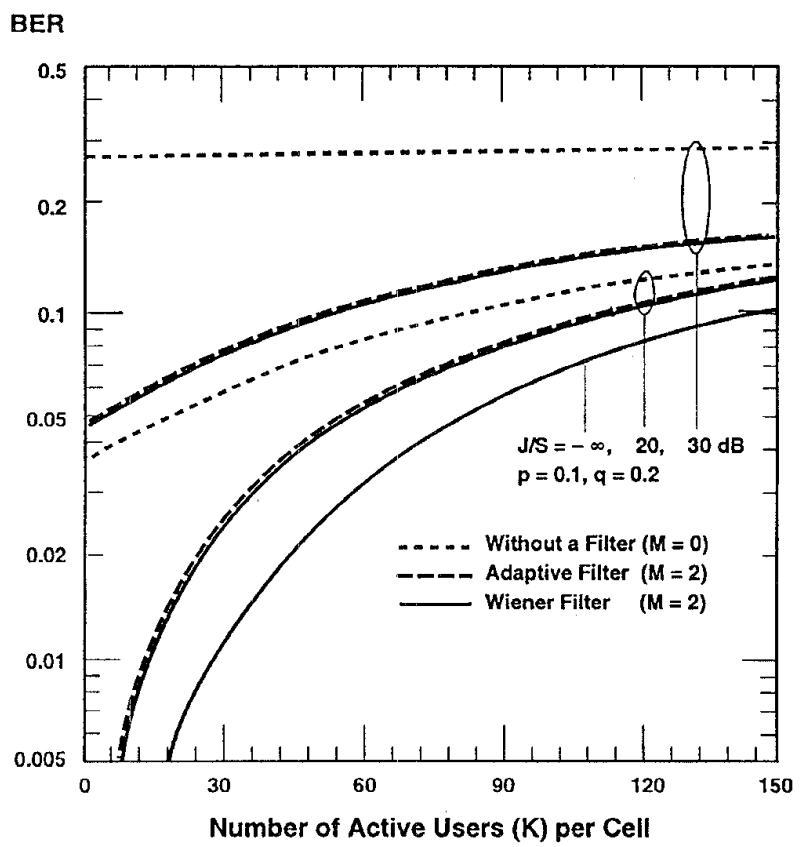

(b)

Fig. 4. The asymptotic BER of CDMA overlay system as a function of the number of active users ( $K$ ) each cell, (a) $q=0$ and (b) $q=0.2$.

significantly worse than is the performance of an ideal Wiener filter, assuming the LMS filter has had sufficient time to converge. Further, the adaptive filter is more effective when the carrier frequency of the narrowband interference is offset from the carrier of the spread spectrum signals. Note that the results shown here do not include diversity and channel coding. Using multipath diversity and interleaved coding, the BER performance can be significantly improved.

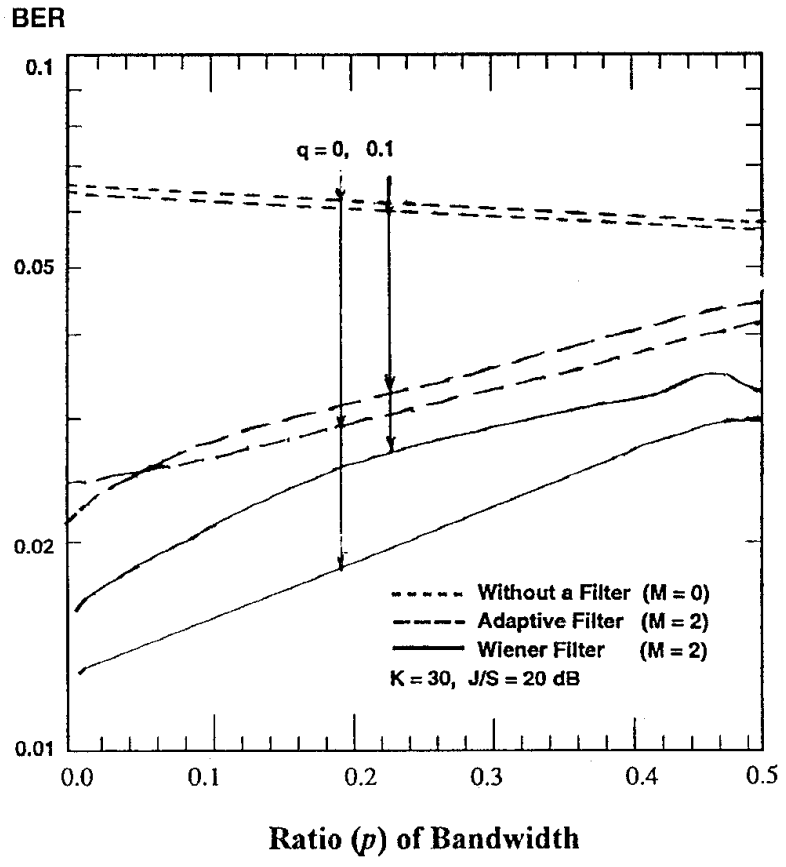

(a)

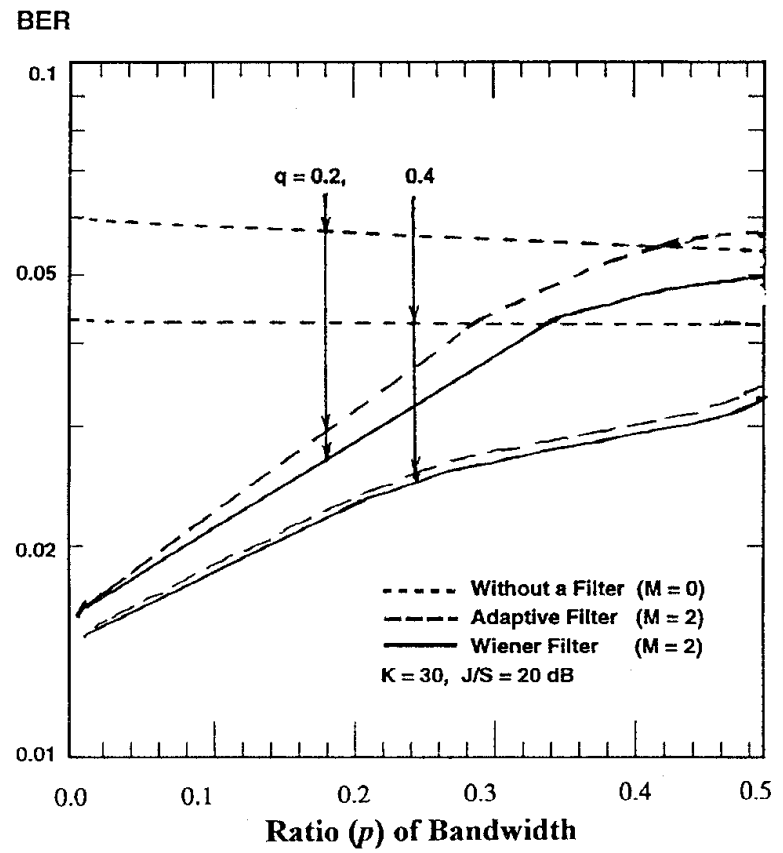

(b)

Fig. 5. The asymptotic BER of CDMA overlay system as a function of the ratio $(p)$ of the interference bandwidth (BW) to spread spectrum $B W$ : (a) $q=0,0.1$ and (b) $q=0.2,0.4$.

\section{APPENDIX A}

DERIVATION OF THE STEADY-STATE TAP WEIGHT COVARIANCE MATRIX OF THE MisadJUSTMENT FILTER

From the adaptive LMS algorithm, the tap weight vector of the misadjustment filter (or the tap error-weight vector of the 


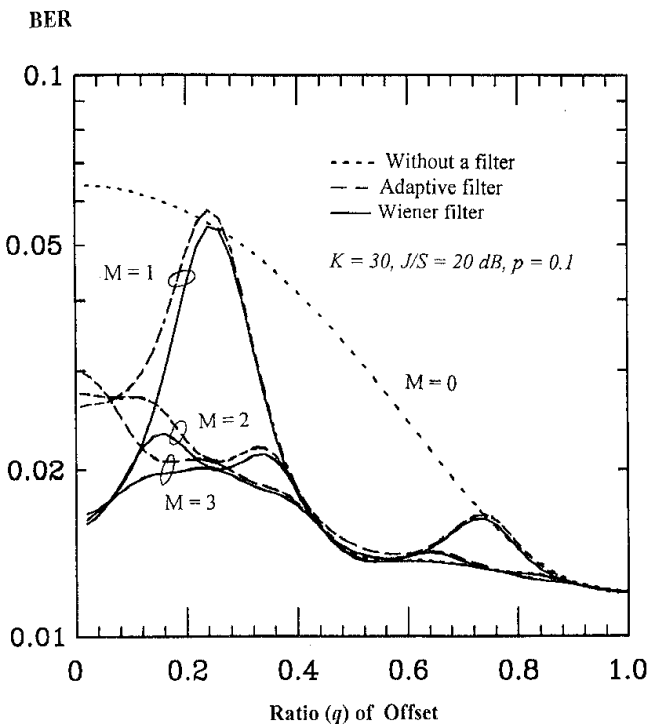

Fig. 6. BER's as a function of the ratio $(q)$ of the interference carrier frequency to the half spread spectrum BW.

adaptive filter) can be presented as [4]

$$
V(j+1)=\left[I-2 \mu X(j) X^{T}(j)\right] V(j)+2 \mu e^{*}(j) X(j)
$$

where $V(j)$ is the column tap weight vector on the $j$ th adaptation, $I$ stands for an identity matrix, $X(j)$ is the sample vector of the input signal on the $j$ th adaptation, $\mu$ is the LMS algorithm step size, and $e^{*}(j)$ is the prediction error at the Wiener filter output on the $j$ th adaptation, and is assumed independent of $V(j)$.

Therefore, the steady-state tap weight covariance matrix can be expressed as

$$
\begin{aligned}
R_{v}= & E\left[V V^{T}\right]=\lim _{j \rightarrow \infty} E\left[V(j+1) V^{T}(j+1)\right] \\
= & \lim _{j \rightarrow \infty} E\left[\left(I-2 \mu X(j) X^{T}(j)\right) V(j) V^{T}(j)\right. \\
& \left.\cdot\left(I-2 \mu X(j) X^{T}(j)\right)\right] \\
& +(2 \mu)^{2} E\left[\left(e^{*}(j)\right)^{2} X(j) X^{T}(j)\right]
\end{aligned}
$$

where it is assumed that the successive input signal vectors $X(j)$ and $X(j+1)$ are independent from adaptation time to adaptation time. In order to approximately guarantee this independence, the period of adaptation time should be at least as great as the correlation time of the narrowband interference. Because the tap weight vector $V(j+1)$ is a function only of the past input signal vectors, $X(j), X(j-1), \cdots, X(0), V(j)$ is independent of $X(j)$. That is

$$
E\left[V(j) X^{T}(j)\right]=E[V(j)] E\left[X^{T}(j)\right]=0 .
$$

The estimate of $V(j)$ is asymptotically unbiased (i.e., $E[V(j)]=0$ ), so that the cross terms in (A2) drop out. Further, (A2) can be decomposed as

$$
\begin{aligned}
R_{v}= & E\left(V V^{T}\right)=E\left(V V^{T}\right)-2 \mu E\left(X X^{T}\right) E\left(V V^{T}\right) \\
& -2 \mu E\left(V V^{T}\right) E\left(X X^{T}\right) \\
& +4 \mu^{2} E\left(X X^{T} V V^{T} X X^{T}\right)+4 \mu^{2} E\left[\left(e^{*}\right)^{2} X X^{T}\right] .
\end{aligned}
$$

Note that although $e^{*}$ and $X$ are uncorrelated by the orthogonality principle, they are not independent, except if the input is Gaussian.

If we express the Wiener prediction error as $e^{*}=x_{0}-$ $\sum_{n=1}^{2 M} \alpha_{n} x_{n}$, where $x_{0}$ represents the current value on the center tap of the Wiener filter (for simplicity, we are indexing from one to $2 M$ instead of from $M$ to $M$, excluding zero), the elements $\left(R_{v}\right)_{m_{1} m_{2}}$ of the asymptotic tap-weight covariance matrix $R_{v}$ satisfy

$$
\begin{aligned}
\left(R_{v}\right)_{m_{1} m_{2}}= & \left(R_{v}\right)_{m_{1} m_{2}}-2 \mu \sum_{n_{1}=1}^{2 M}\left(R_{r}\right)_{m_{1} n_{1}}\left(R_{v}\right)_{n_{1} m_{2}} \\
& -2 \mu \sum_{n_{1}=1}^{2 M}\left(R_{v}\right)_{m_{1} n_{1}}\left(R_{r}\right)_{n_{1} m_{2}} \\
& +4 \mu^{2} \sum_{n_{1}=1}^{2 M} \sum_{n_{2}=1}^{2 M}\left(R_{v}\right)_{n_{1} n_{2}} E\left(x_{m_{1}} x_{m_{2}} x_{n_{1}} x_{n_{2}}\right) \\
& +4 \mu^{2} E\left\{\left[x_{0}^{2}-2 x_{0} \sum_{n_{1}=1}^{2 M} \alpha_{n_{1}} x_{n_{1}}\right.\right. \\
& \left.\left.\left.+\sum_{n_{1}=1}^{2 M} \sum_{n_{2}=1}^{2 M} \alpha_{n_{1}} \alpha_{n_{2}} x_{n_{1}} x_{n_{2}}\right] x_{m_{1}} x_{m_{2}}\right\} \quad \text { (A5 }\right)
\end{aligned}
$$

where $R_{r}$ is the covariance matrix of the input sample vector. Then, (A5) becomes

$$
\begin{aligned}
\sum_{n_{1}=1}^{2 M}\left[\left(R_{r}\right)_{m_{1} n_{1}}\left(R_{v}\right)_{n_{1} m_{2}}+\left(R_{v}\right)_{m_{1} n_{1}}\left(R_{r}\right)_{n_{1} m_{2}}\right] \\
=2 \mu \sum_{n_{1}=1}^{2 M} \sum_{n_{2}=1}^{2 M}\left(R_{v}\right)_{n_{1} n_{2}} E\left(x_{m_{1}} x_{m_{2}} x_{n_{1}} x_{n_{2}}\right) \\
+2 \mu E\left\{\left[x_{0}^{2}-2 x_{0} \sum_{n_{1}=1}^{2 M} \alpha_{n_{1}} x_{n_{1}}\right.\right. \\
\left.\left.+\sum_{n_{1}=1}^{2 M} \sum_{n_{2}=1}^{2 M} \alpha_{n_{1}} \alpha_{n_{2}} x_{n_{1}} x_{n_{2}}\right] x_{m_{1}} x_{m_{2}}\right\} .
\end{aligned}
$$

The solution of (A6) is a function of $\mu$, so that the first term on the right hand side (rhs) is a function of $\mu^{2}$. When $\mu$ is selected sufficiently small in steady state, the first term on the rhs can be neglected, and we have

$$
\begin{aligned}
\sum_{n_{1}=1}^{2 M}[ & \left.\left(R_{r}\right)_{m_{1} n_{1}}\left(R_{v}\right)_{n_{1} m_{2}}+\left(R_{v}\right)_{m_{1} n_{1}}\left(R_{r}\right)_{n_{1} m_{2}}\right] \\
\approx & 2 \mu E\left\{\left[x_{0}^{2}-2 x_{0} \sum_{n_{1}=1}^{2 M} \alpha_{n_{1}} x_{n_{1}}\right.\right. \\
& \left.\left.+\sum_{n_{1}=1}^{2 M} \sum_{n_{2}=1}^{2 M} \alpha_{n_{1}} \alpha_{n_{2}} x_{n_{1}} x_{n_{2}}\right] x_{m_{1}} x_{m_{2}}\right\} .
\end{aligned}
$$

If we assume initially that the input signal is Gaussian, $e^{*}$ is independent of $X$. Therefore, (A7) becomes

$$
\begin{array}{r}
\sum_{n_{1}=1}^{2 M}\left[\left(R_{r}\right)_{m_{1} n_{1}}\left(R_{v}\right)_{n_{1} m_{2}}+\left(R_{v}\right)_{m_{1} n_{1}}\left(R_{r}\right)_{n_{1} m_{2}}\right] \\
\approx 2 \mu E\left[\left(e^{*}\right)^{2}\right]\left(R_{r}\right)_{m_{1} m_{2}}
\end{array}
$$


and (A8) can be represented as

$$
R_{r} R_{v}+R_{v} R_{r} \approx 2 \mu E\left[\left(e^{*}\right)^{2}\right] R_{r}
$$

Equation (A9) may be uncoupled using the eigenvalue--eigenvector decomposition

$$
\begin{aligned}
& R_{r}=Q \Lambda Q^{-1} \\
& R_{v}=Q \hat{R}_{v} Q^{-1}
\end{aligned}
$$

where $Q$ and $Q^{-1}$ are an orthogonal (real unitary) matrix of eigenvectors and its inverse matrix, respectively, $\Lambda$ is a diagonal matrix of the eigenvalues of $R_{r}$ and $\hat{R}_{v}$ is the weight covariance matrix in the principal axis system. Using this transformation, (A9) can be expressed as

$Q \Lambda Q^{-1} Q \hat{R}_{v} Q^{-1}+Q \hat{R}_{v} Q^{-1} Q \Lambda Q^{-1} \approx 2 \mu E\left[\left(e^{*}\right)^{2}\right] Q \Lambda Q^{-1}$

or

$$
\Lambda \hat{R}_{v}+\hat{R}_{v} \Lambda \approx 2 \mu E\left[\left(e^{*}\right)^{2}\right] \Lambda
$$

Because $\hat{R}_{v}$ is symmetric, (A1la) reduces to

$$
\begin{array}{r}
\left(\lambda_{i}+\lambda_{j}\right) a_{i j}=0 \text { for } i \neq j \\
a_{i i}=\mu E\left[\left(e^{*}\right)^{2}\right], \quad \text { for } i=1, \cdots, 2 M
\end{array}
$$

where $\lambda_{i}$ are the eigenvalues of $R_{r}$ and $a_{i j}$ is the $i$ th row and the $j$ th column element of $\hat{R}_{v}$. Again, because $R_{r}$ is a correlation matrix, the eigenvalues $\lambda_{i}$ of $R_{r}$ are always greater than or equal to zero. Assuming that none of the $\lambda_{i}$ equal zero, $a_{i j}=0$ for $i \neq j$. That is, $\hat{R}_{v}$ is a diagonal matrix. Because both $\Lambda$ and $\hat{R}_{v}$ are diagonal, $\Lambda \hat{R}_{v}=\hat{R}_{v} \Lambda$. Therefore, (A11a) reduces to

$$
\hat{R}_{v} \approx \mu E\left[\left(e^{*}\right)^{2}\right] I
$$

From (A10b) and (A12), we have

$$
R_{v}=Q \hat{R}_{v} Q^{-1} \approx \mu E\left[\left(e^{*}\right)^{2}\right] I .
$$

With the Gaussian assumption of the input signal, the tap weight covariance matrix is a diagonal matrix, which completely defines the statistics of the misadjustment filter for the Gaussian input signal. That is, in the steady state, the variance of different tap weights are equal and different tap weights are uncorrelated and independent.

Since our input is not Gaussian, it will be seen that the tap weight covariance matrix is no longer diagonal. We begin the derivation of the tap-weight covariance matrix by considering some properties of the input signal. Define the input signal as

$$
x_{m}=n_{m}+j_{m}
$$

where $n_{m}$ and $j_{m}$ stand for Gaussian noise (both white noise and CDMA signals) and narrowband interference, respectively, and $n_{m}$ and $j_{m}$ are independent.

The first and second moments of the narrowband interference samples are identical to those that would result if $j_{m}$ were samples of a Gaussian process. However, the fourth moment of $x_{m}$ is given by

$$
\begin{aligned}
E\left[x_{1} x_{2} x_{3} x_{4}\right] & \\
= & E\left[\left(n_{1}+j_{1}\right)\left(n_{2}+j_{2}\right)\left(n_{3}+j_{3}\right)\left(n_{4}+j_{4}\right)\right] \\
= & E\left(n_{1} n_{2} n_{3} n_{4}\right)+E\left(n_{1} n_{2} n_{3} j_{4}\right)+E\left(n_{1} n_{2} n_{4} j_{3}\right) \\
& +E\left(n_{1} n_{2} j_{3} j_{4}\right)+E\left(n_{1} n_{3} n_{4} j_{2}\right)+E\left(n_{1} n_{3} j_{2} j_{4}\right) \\
& +E\left(n_{1} n_{4} j_{2} j_{3}\right)+E\left(n_{1} j_{2} j_{3} j_{4}\right)+E\left(n_{2} n_{3} n_{4} j_{1}\right) \\
& +E\left(n_{2} n_{3} j_{1} j_{4}\right)+E\left(n_{2} n_{4} j_{1} j_{3}\right)+E\left(n_{2} j_{1} j_{3} j_{4}\right) \\
& +E\left(n_{3} n_{4} j_{1} j_{2}\right)+E\left(n_{3} j_{1} j_{2} j_{4}\right) \\
& +E\left(n_{4} j_{1} j_{2} j_{3}\right)+E\left(j_{1} j_{2} j_{3} j_{4}\right) .
\end{aligned}
$$

Since

$$
\begin{aligned}
E\left(n_{1} n_{2} n_{3} j_{4}\right) & =E\left(n_{1} n_{2} n_{4} j_{3}\right)=E\left(n_{1} n_{3} n_{4} j_{2}\right) \\
& =E\left(n_{1} j_{2} j_{3} j_{4}\right)=0
\end{aligned}
$$

and

$$
\begin{aligned}
E\left(n_{2} n_{3} n_{4} j_{1}\right) & =E\left(n_{2} j_{1} j_{3} j_{4}\right)=E\left(n_{3} j_{1} j_{2} j_{4}\right) \\
& =E\left(n_{4} j_{1} j_{2} j_{3}\right)=0
\end{aligned}
$$

(A15) reduces to

$$
\begin{aligned}
E\left(x_{1} x_{2} x_{3} x_{4}\right) & \\
= & E\left(n_{1} n_{2} n_{3} n_{4}\right)+E\left(n_{1} n_{2}\right) E\left(j_{3} j_{4}\right)+E\left(n_{1} n_{3}\right) E\left(j_{2} j_{4}\right) \\
& +E\left(n_{1} n_{4}\right) E\left(j_{2} j_{3}\right)+E\left(n_{2} n_{3}\right) E\left(j_{1} j_{4}\right) \\
& +E\left(n_{2} n_{4}\right) E\left(j_{1} j_{3}\right)+E\left(n_{3} n_{4}\right) E\left(j_{1} j_{2}\right)+E\left(j_{1} j_{2} j_{3} j_{4}\right) \\
= & \left.E\left(x_{1} x_{2} x_{3} x_{4}\right)\right|_{\text {Gaussian }}+E\left(j_{1} j_{2} j_{3} j_{4}\right) \\
& -\left.E\left(j_{1} j_{2} j_{3} j_{4}\right)\right|_{\text {Gaussian }}
\end{aligned}
$$

where $\left.E\left(x_{1} x_{2} x_{3} x_{4}\right)\right|_{\text {Gaussian }}$ is defined as $E\left(x_{1} x_{2} x_{3} x_{4}\right)$ under the assumption that $x_{1}, x_{2}, x_{3}, x_{4}$ are jointly Gaussian. By substituting the expression for the fourth moment of the input signal into (A7), we obtain the following relation for the elements of the tap-weight error vector covariance matrix in steady state:

$$
\begin{aligned}
\sum_{n_{1}=1}^{2 M}[( & \left.\left.R_{r}\right)_{m_{1} n_{1}}\left(R_{v}\right)_{n_{1} m_{2}}+\left(R_{v}\right)_{m_{1} n_{1}}\left(R_{r}\right)_{n_{1} m_{2}}\right] \\
\approx & 2 \mu E\left\{\left[x_{0}^{2}-2 x_{0} \sum_{n_{1}=1}^{2 M} \alpha_{n_{1}} x_{n_{1}}\right.\right. \\
& \left.\left.+\sum_{n_{1}=1}^{2 M} \sum_{n_{2}=1}^{2 M} \alpha_{n_{1}} \alpha_{n_{2}} x_{n_{1}} x_{n_{2}}\right] x_{m_{1}} x_{m_{2}}\right\}\left.\right|_{\text {Gaussian }} \\
& +2 \mu\left\{E\left(j_{0}^{2} j_{m_{1}} j_{m_{2}}\right)-\left.E\left(j_{0}^{2} j_{m_{1}} j_{m_{2}}\right)\right|_{\text {Gaussian }}\right\} \\
& -4 \mu \sum_{n_{1}=1}^{2 M} \alpha_{n_{1}}\left\{E\left(j_{0} j_{n_{1}} j_{m_{1}} j_{m_{2}}\right)\right. \\
& \left.-\left.E\left(j_{0} j_{n_{1}} j_{m_{1}} j_{m_{2}}\right)\right|_{\text {Gaussian }}\right\} \\
& +2 \mu \sum_{n_{1}=1}^{2 M} \sum_{n_{2}=1}^{2 M} \alpha_{n_{1}} \alpha_{n_{2}}\left\{E\left(j_{n_{1}} j_{n_{2}} j_{m_{1}} j_{m_{2}}\right)\right. \\
& \left.-\left.E\left(j_{n_{1}} j_{n_{2}} j_{m_{1}} j_{m_{2}}\right)\right|_{\text {Gaussian }}\right\}
\end{aligned}
$$




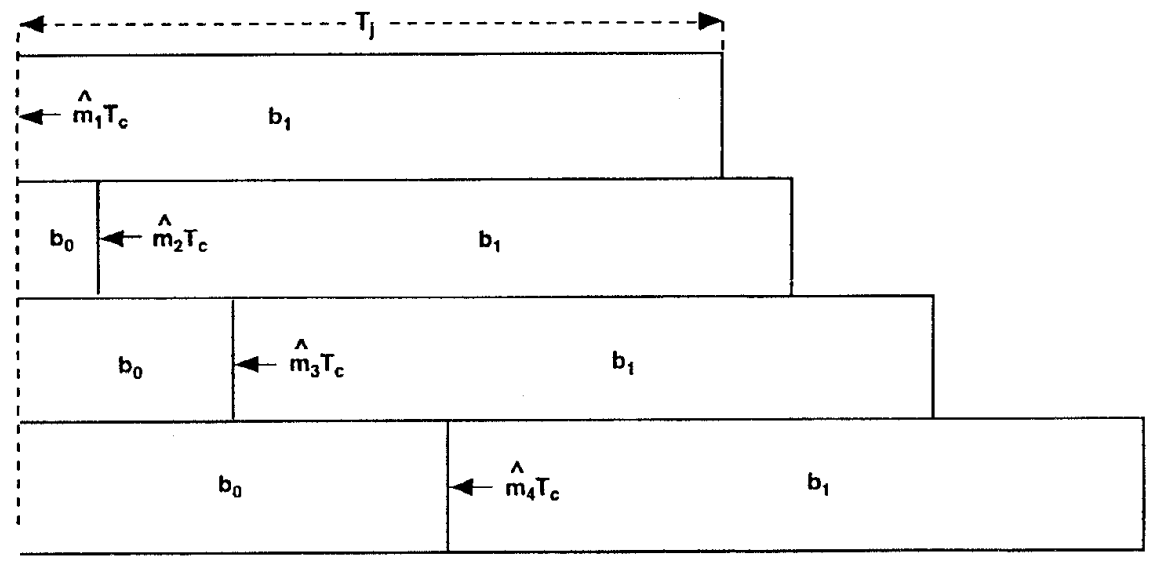

Fig. 7. Bit sequence and its delayed positions needed in the evaluation of the fourth moment ( $b_{0}$ and $b_{1}$ are two independent adjacent bits).

or

$$
\begin{aligned}
\sum_{n_{1}=1}^{2 M}[ & \left.\left(R_{r}\right)_{m_{1} n_{1}}\left(R_{v}\right)_{n_{1} m_{2}}+\left(R_{v}\right)_{m_{1} n_{1}}\left(R_{r}\right)_{n_{1} m_{2}}\right] \\
\approx & 2 \mu E\left[\left(e^{*}\right)^{2}\right]\left(R_{r}\right)_{m_{1} m_{2}}+2 \mu E\left[\left(e_{j}^{*}\right)^{2} j_{m_{1}} j_{m_{2}}\right] \\
& -2 \mu E\left[\left(e_{j}^{*}\right)^{2}\right] E\left(j_{m_{1}} j_{m_{2}}\right)
\end{aligned}
$$

where

$$
e_{j}^{*}=j_{0}-\sum_{n_{1}=1}^{2 M} \alpha_{n_{1} j_{n_{1}}}
$$

denotes the narrowband interference component of the Wiener prediction error. In order to solve equation (A16), we must know the second and the fourth moments of the BPSK narrowband interference. The second moment of the narrowband interference term in (1) is given by

$$
\begin{aligned}
& E\left(j_{m_{1}} j_{m_{2}}\right) \\
& \quad=E\left[J\left(t+m_{1} T_{c}\right) J\left(t+m_{2} T_{c}\right)\right] \\
& \quad=J \operatorname{rect}\left(1-\left|m_{1}-m_{2}\right| T_{c} / T_{j}\right) \cos \left[2 \pi\left(m_{1}-m_{2}\right) \Delta T_{c}\right] \\
& \quad=J \operatorname{rect} \cdot\left(1-\left|m_{1}-m_{2}\right| p\right) \cos \left[2 \pi\left(m_{1}-m_{2}\right) q\right] . \quad \text { (A20) }
\end{aligned}
$$
by

The fourth moment of the narrowband interference is given

$$
\begin{aligned}
E\left[j_{m_{1}}\right. & \left.j_{m_{2}} j_{m_{3}} j_{m_{4}}\right] \\
= & (2 J)^{2} E\left\{d\left(t+m_{1} T_{c}\right) \cos \left[2 \pi\left(f_{0}+\Delta\right)\left(t+m_{1} T_{c}\right)+\theta\right]\right. \\
& \cdot d\left(t+m_{2} T_{c}\right) \cos \left[2 \pi\left(f_{0}+\Delta\right)\left(t+m_{2} T_{c}\right)+\theta\right] \\
& \cdot d\left(t+m_{3} T_{c}\right) \cos \left[2 \pi\left(f_{0}+\Delta\right)\left(t+m_{3} T_{c}\right)+\theta\right] \\
& \left.\cdot d\left(t+m_{4} T_{c}\right) \cos \left[2 \pi\left(f_{0}+\Delta\right)\left(t+m_{4} T_{c}\right)+\theta\right]\right\} \\
= & \left(J^{2} / 2\right) E\left[d\left(t+m_{1} T_{c}\right) d\left(t+m_{2} T_{c}\right)\right. \\
& \left.\cdot d\left(t+m_{3} T_{c}\right) d\left(t+m_{4} T_{c}\right)\right] \\
& \cdot\left\{\cos \left[2 \pi\left(m_{1}+m_{2}-m_{3}-m_{4}\right) q\right]\right. \\
& +\cos \left[2 \pi\left(m_{1}-m_{2}+m_{3}-m_{4}\right) q\right] \\
& \left.+\cos \left[2 \pi\left(m_{1}-m_{2}-m_{3}+m_{4}\right) q\right]\right\}
\end{aligned}
$$

where $E\left[d\left(t+m_{1} T_{c}\right) d\left(t+m_{2} T_{c}\right) d\left(t+m_{3} T_{c}\right) d\left(t+m_{4} T_{c}\right)\right]$ is the fourth moment of the binary sequence, $j(t)$. As shown in Fig. $7, d\left(t+m_{1} T_{c}\right) d\left(t+m_{2} T_{c}\right) d\left(t+m_{3} T_{c}\right) d\left(t+m_{4} T_{c}\right)$ depends on both the random position and the value of $j(t)$ at any instant of time. Assuming $2 M T_{c}<T_{j}$, one obtains

$$
\begin{aligned}
& E\left[d\left(t+m_{1} T_{c}\right) d\left(t+m_{2} T_{c}\right) d\left(t+m_{3} T_{c}\right) d\left(t+m_{4} T_{c}\right)\right] \\
& \quad=\operatorname{rect}\left(1-\left|\hat{m}_{1}-\hat{m}_{4}\right| T_{c} / T_{j}+\left|\hat{m}_{2}-\hat{m}_{3}\right| T_{c} / T_{j}\right) \\
& \quad=\operatorname{rect}\left(1-\left|\hat{m}_{1}-\hat{m}_{4}\right| p+\left|\hat{m}_{2}-\hat{m}_{3}\right| p\right)
\end{aligned}
$$

where $\left\{\hat{m}_{1}, \hat{m}_{2}, \hat{m}_{3}, \hat{m}_{4}\right\}=\left\{m_{1}, m_{2}, m_{3}, m_{4}\right\}$ and $\hat{m}_{4} \geq$ $\hat{m}_{3} \geq \hat{m}_{2} \geq \hat{m}_{1}$.

\section{APPENDIX $\underset{\substack{C K \\ k=1 \\ k \leq i}}{\mathrm{~B}} I_{k}=\sum_{\substack{k=1 \\ k \leq i}}^{C K} \sum_{\hat{l}=1}^{L} I_{k, \hat{l}}$}

$I_{k, \hat{l}}$ can be written as

$$
\begin{array}{r}
I_{k, \hat{l}}=\sqrt{2 P}\left[A_{k \hat{l}} \cos \left(\phi_{k \hat{l}}\right)+\beta_{k \hat{l}} \cos \left(\psi_{k i}\right)\right] \\
\cdot T_{c} \sqrt{\epsilon\left(\gamma, c_{k}, k\right)} \hat{I}_{k, \hat{l}}
\end{array}
$$

where

$$
\begin{aligned}
\hat{I}_{k, \hat{l}}= & \sum_{m=-M}^{M}\left(\alpha_{m}+v_{m}\right)\left\{b _ { k } ^ { ( \lambda - 1 ) } \left[\tau_{0} \sum_{j=0}^{n} a_{j}^{(i)} a_{j-n-m-1}^{(k)}\right.\right. \\
& \left.+\left(T_{c}-\tau_{0}\right) \sum_{j=0}^{n-1} a_{j}^{(i)} a_{j-n-m}^{(k)}\right] \\
& +b_{k}^{(\lambda)}\left[\tau_{0} \sum_{j=n+1}^{N-1} a_{j}^{(i)} a_{j-n-m-1}^{(k)}\right. \\
& \left.\left.+\left(T_{c}-\tau_{0}\right) \sum_{j=n}^{N-1} a_{j}^{(i)} a_{j-n-m}^{(k)}\right]\right\}
\end{aligned}
$$

and where $n=\operatorname{int}\left[\frac{\tau_{k l}}{T_{c}}\right]$ and $\tau_{0}=\frac{\tau_{k l}}{T_{c}}-n$. The conditional variance of $\hat{I}_{k, \hat{l}}$, conditioned on $\left\{a_{j}^{(i)}\right\},\left\{a_{j}^{(k)}\right\}, \tau_{0}$, and $\left\{v_{m}\right\}$, 
is given by

$$
\begin{aligned}
E\left[\left.\left(\hat{I}_{k, l}\right)^{2}\right|_{\left\{a_{j}^{(k)}\right\},\left\{a_{j}^{(l)}\right\}, \tau_{0},\left\{v_{m_{2}}\right\}}\right] & \sum_{m_{1}=-M} \sum_{m_{2}=-M}^{M}\left(\alpha_{m_{1}}+v_{m_{1}}\right)\left(\alpha_{m_{2}}+v_{m_{2}}\right) \\
& \cdot\left\{\tau_{0}^{2} \sum_{j_{1}=0}^{n} \sum_{j_{2}=0}^{n} a_{j_{1}}^{(i)} a_{j_{2}}^{(i)} a_{j_{1}-n-m_{1}-1}^{(k)} a_{j_{2}-n-m_{2}-1}^{(k)}\right. \\
& +\left(T_{C}-\tau_{0}\right)^{2} \sum_{j_{1}=0}^{n-1} \sum_{j_{2}=0}^{n-1} a_{j_{1}}^{(i)} a_{j_{2}}^{(i)} a_{j_{1}-n-m_{1}}^{(k)} a_{j_{2}-n-m_{2}}^{(k)} \\
& +2\left(T_{c}-\tau_{0}\right) \tau_{0} \\
& \cdot \sum_{j_{1}=0}^{n} \sum_{j_{2}=0}^{n-1} a_{j_{1}}^{(i)} a_{j_{2}}^{(i)} a_{j_{1}-n-m_{1}-1}^{(k)} a_{j_{2}-n-m_{2}}^{(k)} \\
& +\tau_{0}^{2} \sum_{j_{1}=n+1}^{N-1} \sum_{j_{2}=n+1}^{N-1} a_{j_{1}}^{(i)} a_{j_{2}}^{(i)} a_{j_{1}-n-m_{1}-1} a_{j_{2}-n-m_{2}-1} \\
& +\left(T_{C}-\tau_{0}\right)^{2} \sum_{j_{1}=n}^{N-1} \sum_{j_{2}=n}^{N-1} a_{j_{1}}^{(i)} a_{j_{2}}^{(i)} a_{j_{1}-n-m_{1}}^{(k)} a_{j_{2}-n-m_{2}}^{(k)} \\
+ & 2 \tau_{0}\left(T_{c}-\tau_{0}\right) \\
& \left.\cdot \sum_{j_{1}=n+1}^{N-1} \sum_{j_{2}=n}^{N-1} a_{j_{1}}^{(i)} a_{j_{2}}^{(i)} a_{j_{1}-n-m_{1}-1}^{(k)} a_{j_{2}-n-m_{2}}^{(k)}\right\}
\end{aligned}
$$

\section{Therefore}

$$
\begin{aligned}
& E\left[\left.\hat{I}_{k, l}^{2}\right|_{\left\{a_{j}^{(i)}\right\}, \tau_{0},\left\{v_{m}\right\}}\right] \\
& =\tau_{0}^{2}(n+1) \sum_{m=-M}^{M}\left(\alpha_{m}+v_{m}\right)^{2} \\
& +\sum_{m_{1}=-M}^{M} \sum_{\substack{m_{2}=-M \\
m_{2} \neq m_{1}}}^{M}\left(\alpha_{m_{1}}+v_{m_{1}}\right)\left(\alpha_{m_{2}}+v_{m_{2}}\right) \\
& \cdot \tau_{0}^{2} \sum_{j=0}^{n} a_{j}^{(i)} a_{j+\left(m_{2}-m_{1}\right)}^{(i)} \\
& +\left(T_{c}-\tau_{0}\right)^{2} n \sum_{m=-M}^{M}\left(\alpha_{m}+v_{m}\right)^{2} \\
& +\sum_{m_{1}=-M}^{M} \sum_{\substack{m_{2}=-M \\
m_{2} \neq m_{1}}}^{M}\left(\alpha_{m_{1}}+v_{m_{1}}\right)\left(\alpha_{m_{2}}+v_{m_{2}}\right)\left(T_{c}-\tau_{0}\right)^{2} \\
& \cdot \sum_{j=0}^{n-1} a_{j}^{(i)} a_{j+\left(m_{2}-m_{1}\right)} \\
& +2 \tau_{0}\left(T_{c}-\tau_{0}\right) n \sum_{m=-M}^{M}\left(\alpha_{m}+v_{m}\right)\left(\alpha_{m+1}+v_{m+1}\right) \\
& +2 \tau_{0}\left(T_{c}-\tau_{0}\right) \\
& \text {. } \sum_{m_{1}=-M}^{M} \sum_{\substack{m_{2}=-M \\
m_{2} \neq m_{1}}}^{M}\left(\alpha_{m_{1}}+v_{m_{1}}\right)\left(\alpha_{m_{2}}+v_{m_{2}}\right)
\end{aligned}
$$

$$
\begin{aligned}
& \cdot \sum_{j=0}^{n-1} a_{j}^{(i)} a_{j+m_{2}-m_{1}-1}^{(i)} \\
& +\tau_{0}^{2}(N-n-1) \sum_{m=-M}^{M} \alpha_{m}^{2} \\
& +\tau_{0}^{2} \sum_{m_{1}=-M}^{M} \sum_{\substack{m_{2}=-M \\
m_{2} \neq m_{1}}}^{M}\left(\alpha_{m_{1}}+v_{m_{1}}\right)\left(\alpha_{m_{2}}+v_{m_{2}}\right) \\
& \cdot \sum_{j=n+1}^{N-1} a_{j}^{(i)} a_{j+\left(m_{2}-m_{1}\right)}^{(i)} \\
& +\left(T_{c}-T_{0}\right)^{2}(N-n) \sum_{m=-M}^{M}\left(\alpha_{m}+v_{m}\right)^{2} \\
& +\left(T_{c}-\tau_{0}\right)^{2} \sum_{m_{1}=-M}^{M} \sum_{\substack{m_{2}=-M \\
m_{2} \neq m_{1}}}^{M}\left(\alpha_{m_{\mathrm{I}}}+v_{m_{1}}\right)\left(\alpha_{m_{2}}+v_{m_{2}}\right) \\
& \cdot \sum_{j=n}^{N-1} a_{j}^{(i)} a_{j+\left(m_{2}-m_{1}\right)}^{(i)} \\
& +2 \tau_{0}\left(T_{c}-\tau_{0}\right)(N-n-1) \\
& \cdot \sum_{m=-M}^{M}\left(\alpha_{m}+v_{m}\right)\left(\alpha_{m+1}+v_{m+1}\right) \\
& +2 \tau_{0}\left(T_{C}-\tau_{0}\right) \\
& \sum_{m_{1}=-M}^{M} \sum_{\substack{m_{2}=-M \\
m_{2} \neq m_{1}+1}}^{M}\left(\alpha_{m_{1}}+v_{m_{1}}\right)\left(\alpha_{m_{2}}+v_{m_{2}}\right) \\
& \cdot \sum_{j=n+1}^{N-1} a_{j}^{(i)} a_{j+m_{2}-m_{1}-1}^{(i)} \\
& =N \tau_{0}^{2} \sum_{m=-M}^{M}\left(\alpha_{m}+v_{m}\right)^{2} \\
& +\tau_{0}^{2} \sum_{m_{1}=-M}^{M} \sum_{\substack{m_{2}=-M \\
m_{2} \neq m_{1}}}^{M}\left(\alpha_{m_{1}}+v_{m_{1}}\right)\left(\alpha_{m_{2}}+v_{m_{2}}\right) \\
& \cdot \sum_{j=0}^{N-1} a_{j}^{(i)} a_{j+m_{2}-m_{1}}^{(i)} \\
& +N\left(T_{c}-\tau_{0}\right)^{2} \sum_{m=-M}^{M}\left(\alpha_{m}+v_{m}\right)^{2} \\
& +\left(T_{c}-\tau_{0}\right)^{2} \\
& \cdot \sum_{m_{1}=-M}^{M} \sum_{\substack{m_{2}=-M \\
m_{2} \neq m_{1}+1}}^{M}\left(\alpha_{m_{1}}+v_{m_{1}}\right)\left(\alpha_{m_{2}}+v_{m_{2}}\right) \\
& \cdot \sum_{j=0}^{N-1} a_{j}^{(i)} a_{j+m_{2}-m_{1}}^{(i)} \\
& +2(N-1) \tau_{0}\left(T_{c}-\tau_{0}\right) \\
& \cdot \sum_{m=-M}^{M}\left(\alpha_{m}+v_{m}\right)\left(\alpha_{m+1}+v_{m+1}\right) \\
& +2 \tau_{0}\left(T_{c}-\tau_{0}\right)
\end{aligned}
$$




$$
\begin{aligned}
& \cdot \sum_{m_{1}=-M}^{M} \sum_{\substack{m_{2}=-M \\
m_{2} \neq m_{1}+1}}^{M}\left(\alpha_{m_{1}}+v_{m_{1}}\right)\left(\alpha_{m_{2}}+v_{m_{2}}\right) \\
& \cdot \sum_{\substack{j=0 \\
j \neq n}}^{N-1} a_{j}^{(i)} a_{j+m_{2}-m_{1}-1}^{(i)} .
\end{aligned}
$$

Note that for large processing gains, terms roughly of the form $\frac{1}{N} \sum_{j=0}^{N-1} a_{j}^{(i)} a_{j+j_{1}}^{(i)}$, for $j_{1} \neq 0, \bmod N$, are approximately zero, since they correspond to an out-of-phase correlation of the spreading sequence. Therefore, we can approximate

$$
\begin{aligned}
& E\left[\left.\left(\hat{I}_{k, \hat{l}}\right)^{2}\right|_{\left\{a_{j}^{(8)}\right\}, \tau_{0},\left\{v_{m}\right\}}\right] \\
& \approx N \tau_{0}^{2} \sum_{m=-M}^{M}\left(\alpha_{m}+v_{m}\right)^{2} \\
& \quad+N\left(T_{c}-\tau_{0}\right)^{2} \sum_{m=-M}^{M}\left(\alpha_{m}+v_{m}\right)^{2} \\
& \quad+2(N-1) \tau_{0}\left(T_{c}-\tau_{0}\right) \\
& \quad \cdot \sum_{m=-M}^{M}\left(\alpha_{m}+v_{m}\right)\left(\alpha_{m+1}+v_{m+1}\right)
\end{aligned}
$$

and thus

$$
\begin{aligned}
& E\left[\left.\left(\hat{I}_{k, \hat{l}}\right)^{2}\right|_{\left\{a_{j}^{(i)}\right\},\left\{v_{m}\right\}}\right] \\
& \approx\left[\sum_{m=-M}^{M}\left(\alpha_{m}+v_{m}\right)^{2}\right] \frac{2 N}{3} \\
& \quad+\left[\sum_{m=-M}^{M}\left(\alpha_{m}+v_{m}\right)\left(\alpha_{m+1}+v_{m+1}\right)\right] \frac{N}{3}
\end{aligned}
$$

Note that $E\left[\left.\left(\hat{I}_{k, l}\right)^{2}\right|_{\left\{a_{j}^{(i)}\right\},\left\{v_{m}\right\}}\right]$ is not a function of $\left\{a_{j}^{(i)}\right\}$. Finally

$$
\begin{aligned}
E & {\left[\left.\left(\sum_{\substack{k=1 \\
k \leq i}}^{C K} I_{k}\right)^{2}\right|_{\left\{a_{j}^{(i)}\right\},\left\{v_{m}\right\}}\right] } \\
= & E\left[\left.\left(\sum_{\substack{k=1 \\
k \leq i}}^{C K} \sum_{\hat{l}=1}^{L} I_{k, \hat{l}}\right)^{2}\right|_{\left\{v_{m}\right\}}\right] \\
= & 2 P\left(A^{2} / 2+\rho\right) T_{c}^{2} \sum_{\substack{k=1 \\
k \leq i}}^{C K} \sum_{\substack{l=1 \\
k=1}}^{L} E\left[\epsilon\left(\gamma, c_{k}, k\right)\right] \\
& \cdot E\left[\left.\left(\hat{I}_{k, i}\right)^{2}\right|_{\substack{\left\{a_{j}^{(i)}\right\},\left\{v_{m}\right\} \\
j}}\right.
\end{aligned}
$$

$$
\begin{aligned}
= & 4[(1+\zeta(\gamma)) K L-L] P\left(A^{2} / 2+\rho\right) T_{b}^{2} \\
& \cdot\left[\sum_{m=-M}^{M}\left(\alpha_{m}+v_{m}\right)^{2}\right. \\
& \left.+\frac{1}{2} \sum_{m=-M}^{M}\left(\alpha_{m}+v_{m}\right)\left(\alpha_{m+1}+v_{m+1}\right)\right] /
\end{aligned}
$$

\section{REFERENCES}

[1] J. Wang and L. B. Milstein, "CDMA overlay situations for microcellular mobile communications," IEEE Trans. Commun. vol. 43, pp. 603-614, Feb. 1995.

[2] R. A. Iltis and L. B. Milstein, "An approximate statistical analysis of the Widrow LMS algorithm with application to narrowband interference rejection," IEEE Trans. Commun., vol. 33, pp. 121-130, Feb. 1985.

[3] N. J. Bershad, "Error probabilities for DS spread spectrum systems using an ALE for narrowband interference rejection," IEEE Trans. Commun. vol. 36, pp. 586-595, May 1988.

[4] B. Widrow and S. D. Stearns, Adaptive Signal Processing. Englewood Cliffs, NJ: Prentice-Hall, 1985.

[5] E. Masry, "Closed form result for the rejection of narrowband interference in PN spread spectrum systems-Part I: Linear prediction filters," IEEE Trans. Commun., vol. COM-32, pp. 888-896, Aug. 1984.

[6] D. A. S. Fraser, Nonparametric Methods in Statistics. New York: Wiley 1957.

[7] G. J. Saulnier, "Suppression of narrowband jammers in a spreadspectrum receiver using transform domain adaptive filtering," IEEE J. Select. Areas Commun., vol. 10, pp. 742-749, May 1992.

[8] G. L. Stuber and C. K. Chao, "Analysis of a multiple cell direct sequence CDMA cellular mobile radio system," IEEE J, Select. Areas Commun., vol. 10, pp. 669-679, May 1992.

[9] R. L. Pickholtz, L. B. Milstein, and D. L. Schilling, "Spread spectrum for mobile communications," IEEE Trans. Veh. Technol., pp. 313-322, May 1991.

[10] L. B. Milstein et al, "On the feasibility of a CDMA overlay for personal communications networks," IEEE J. Select. Areas Commun., vol. 10, pp. 655-668, May 1992.

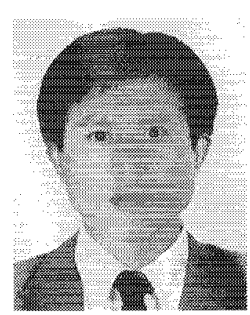

Jiangzhou Wang (M'91-SM'94) was born in Hubei, China, on November 15, 1961. He received the B.S. and M.S. degrees from Xidian University, Xian, China, in 1983 and 1985 , respectively, and the Ph.D. degree (with Greatest Distinction) from the University of Ghent, Belgium, in 1990, all in electrical engineering.

From 1990 to 1992 , he was a Postdoctoral Fellow in the University of California at San Diego, where he worked on the research and development of cellular CDMA systems. From 1992 to 1995 , he was a Senior System Engineer at Rockwell Corporation, Newport Beach, $\mathrm{CA}$, where he worked on the development and system design of wireless communications. Since 1995, he has been with the University of Hong Kong, where he is currently an Associate Professor. He is teaching and conducting research in the areas of cellular mobile and spread spectrum communications. He holds one U.S. patent.

Laurence B. Milstein (S'66-M'68-SM'77-F'85), for a photograph and biography, see this issue, p. 1499. 